\title{
Prior oral conditions in patients undergoing heart valve surgery
}

\author{
Francisco-Javier Silvestre ${ }^{1}$, Irene Gil-Raga ${ }^{2}$, Mayte Martinez-Herrera ${ }^{3}$, Dorina Lauritano ${ }^{4}$, Javier Silvestre- \\ Rangil ${ }^{5}$
}

${ }^{1}$ Professor, Department od Stomatology, University of Valencia, Spain

${ }^{2}$ Diplomate of medical-surgery dentistry, University of Valencia. Spain

${ }^{3}$ Research fellow, Department of Stomatology, University of Valencia. Spain

${ }^{4}$ Professor of Medicine and Surgery, Department of Universitty of Milano-Bicocca. Italy

${ }^{5}$ Associate Professor, Department of Stomatology, University of Valencia

Correspondence:

Unidad de Estomatología

Hospital Universitario Dr. Peset

C/ Juan de Garay s/n (46017-Valencia. España)

francisco.silvestre@uv.es

\begin{abstract}
Silvestre FJ, Gil-Raga I, Martinez-Herrera M, Lauritano D, SilvestreRangil J. Prior oral conditions in patients undergoing heart valve surgery. J Clin Exp Dent. 2017;9(11):e1287-91.
\end{abstract}

Received: 03/04/2017 Accepted: 28/06/2017

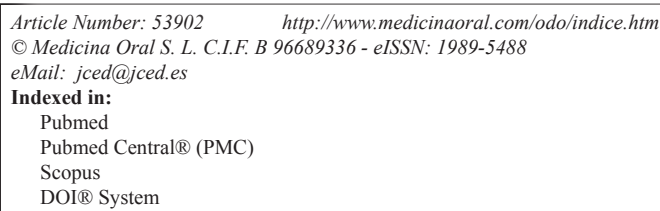

\begin{abstract}
Background: Patients scheduled for heart valve surgery should be free of any oral infectious disorders that might pose a risk in the postoperative period. Few studies have been made on the dental conditions of such patients prior to surgery. The present study describes the most frequent prior oral diseases in this population group.

Material and Methods: A prospective, observational case-control study was designed involving 60 patients (30 with heart valve disease and 30 controls, with a mean age of 71 years in both groups). A dental exploration was carried out, with calculation of the DMFT (decayed, missing and filled teeth) index and recording of the periodontal parameters (plaque index, gingival bleeding index, periodontal pocket depth, and attachment loss). The oral mucosa was also examined, and panoramic X-rays were used to identify possible intrabony lesions.

Results: Significant differences in bacterial plaque index were observed between the two groups $(p<0.05)$, with higher scores in the patients with valve disease. Probing depth and the presence of moderate pockets were also greater in the patients with valve disease than among the controls $(p<0.01)$. Sixty percent of the patients with valve disease presented periodontitis.

Conclusions: Patients scheduled for heart valve surgery should be examined for possible active periodontitis before the operation. Those individuals found to have periodontal disease should receive adequate periodontal treatment before heart surgery.
\end{abstract}

Key words: Valve disease, aortic, mitral, heart surgery, periodontitis.

\section{Introduction}

Heart valve diseases are an important cause of morbidity among elderly people all over the world. In the concrete case of Spain, where the population life expectancy is very long, such diseases become particularly important.
Heart valve disorders comprise a series of congenital and acquired conditions, the underlying causes of which have varied over the last decades (1).

All of the heart valves can suffer alterations due to different etiologies such as myxomatous mitral valve disease 
or senile calcified aortic valve stenosis (2). Less frequent presentations include severe tricuspid valve regurgitation secondary to pacemaker implantation (3). Echocardiography is an important tool that allows the identification of anatomical heart valve changes in patients without a prior diagnosis (4). In this context it is important to consider optimum timing of treatment, without waiting for the symptoms to become serious (5).

Dental infections, and particularly periodontal disease, have been associated to cardiovascular disorders $(6,7)$, though clinical confirmation in such cases is complicated by the influence of common risk factors and confounding factors (8-10). In the preoperative period of cardiovascular surgery, patients should be screened for the possible existence of odontogenic infections caused by dental caries or periodontitis, and which can result in increased morbidity-mortality $(11,12)$. In this regard, patients that have not received preoperative dental treatment might be at increased risk in the postoperative period.

Few studies have been published on the presurgical oral health of patients scheduled for heart valve surgery. Such individuals have been found to have few remaining teeth, with important dental caries and periodontal alterations, though apart from the typically advanced age of such patients, the possible causal factors underlying these alterations have not been established (11-13).

The present study was carried out to identify the most frequent oral disorders in patients during the preoperative period of heart valve surgery.

\section{Material and Methods}

-Study design and population

A prospective, case-control study was designed to determine the oral condition of patients scheduled for heart valve surgery and referred from the Department of Cardiology to the Stomatology Unit of Dr. Peset University Hospital (Valencia, Spain).

The study was approved by the Clinical Research Ethics Committee of the University of Valencia, and written informed consent was obtained from each patient.

We initially selected 33 individuals, though three failed to meet the inclusion criteria and were thus excluded from the study, leaving a final total of 30 patients with a mean age of $71.5 \pm 9.5$ years ( 21 females and 9 males). The included patients were required to be scheduled for heart valve surgery; have no serious systemic disorders other than heart valve disease; and have at least 10 teeth in the mouth.

Sample size calculation was carried out to establish casecontrol comparisons of the quantitative effect variables (plaque index, bleeding index, periodontal pocket depth, etc.) with a statistical power of $80 \%$ and an alpha error of 0.05 , capable of detecting a standardized difference of 0.8 according to the Cohen scale (14). Sample Power 2.0 (SPSS Inc., Chicago, IL, USA) was used to establish a total of 26 patients per group. We raised this figure slightly to 33 individuals per group, with the final inclusion of 30, in prevision of possible missing data.

An age- and gender-matched control group (mean age $71.2 \pm 9.2$ years; 21 females and 9 males) was recruited on a randomized basis from among the people accompanying the patients. Apart from the absence of heart disease (and therefore no need for cardiovascular surgery), the controls were required to meet the same inclusion criteria as the patients.

-Study parameters

The case history was compiled, with the collection of information on general health and a thorough oral examination, with the obtainment of panoramic X-rays in all subjects. The DMFT (decayed, missing and filled teeth) index was recorded with the help of an intraoral dental mirror and probe. All the teeth were assessed for active caries and root fragments; fillings, crowns and bridges; and endodontic treatment. Missing teeth were evaluated clinically and radiologically. The sum of the components of the DMFT scale was divided by the number of teeth in the mouth.

We also recorded periodontal parameters such as plaque index (PI), bleeding index (BI), pocket depth (PD) and clinical attachment loss (CAL). All the mesial and palatine or lingual surfaces of the teeth were evaluated in order to calculate the plaque index, scored from 0-4 (0 $=$ not detectable $;=$ only detectable with the probe; 2 $=$ moderate and visible; $3=$ abundant plaque extending beyond the cervical third of the tooth). The mean score of all the teeth was calculated.

Pocket depth in turn was measured at 6 points per tooth ( 3 buccal and 3 palatine or lingual): a depth of up to 3 $\mathrm{mm}$ was considered physiological; 4-5 mm corresponded to moderate pockets; and $\geq 6 \mathrm{~mm}$ was taken to be indicative of severe periodontal pockets.

The bleeding index was measured by probing at four points, calculating the mean bleeding area per explored surface.

Recession was calculated as the distance (in $\mathrm{mm}$ ) between the cementoenamel junction and the free gingival margin, while clinical attachment loss was determined as the distance (in $\mathrm{mm}$ ) between the cementoenamel junction and the depth of the periodontal pouch.

The oral mucosa was also thoroughly explored in order to detect possible structural changes or anomalies: tongue, lips, check mucosa, gums, palate (hard and soft), floor of the mouth, oropharyngeal isthmus and epiglottis.

Lastly, the panoramic X-rays images were evaluated for possible radiopacities, translucencies or mixed images.

-Statistical analysis

A descriptive analysis was made of the study variables, reporting continuous variables as the mean and standard deviation, and categorical variables as frequency and percentage. The Student t-test for dependent samples 
was used to establish comparisons between the case and control groups. Normal data distribution was previously confirmed using the Kolmogorov-Smirnov test (14). The chi-squared test in turn was used to establish associations between categorical variables.

Due to the limited sample size, the Mann-Whitney Utest was used to contrast parameters according to the type of valve disease involved (mitral or aortic). Statistical significance was considered for $p \leq 0.05$.

\section{Results}

Both groups (case and control) involved the same number of individuals $(n=30)$ and were homogeneous in terms of age and gender distribution (Table 1). re), statistical significance was seen to be limited to moderate pocket depth $(57.9 \pm 25.4 \%$ in the patients versus $39.0 \pm 15.7 \%$ in the controls; $p=0.002$ ).

Gingival recession was greater among the controls than in the patients with valve disease $(1.07 \mathrm{~mm}$ versus 0.91 $\mathrm{mm} ; p=0,552)$, though attachment loss was greater among the latter $(4.49 \pm 1.01 \mathrm{~mm}$ versus $4.15 \mathrm{v} 1.11 \mathrm{~mm}$ in the control group; $p=0.265$ ).

Most of the patients with heart valve disease had no oral mucosal lesions; only two small angiomas and one fibroma of the cheek mucosa were diagnosed. Likewise, the panoramic X-rays revealed no bone lesions.

On dividing the patients according to the type of valve disorder, 16 were seen to suffer aortic valve stenosis $(8$

Table 1: Simple size, age, main caries and periodontal parameters measured between the group of patients undergoing heart valve surgery and control group.

\begin{tabular}{|l|c|c|c|}
\hline & Controls & Cases & $\boldsymbol{p}$ \\
\hline n (females) & $30(21)$ & $30(21)$ & \\
Age (years) & $71,2 \pm 9,2$ & $71,5 \pm 9,5$ & \\
DMFT & $14,8 \pm 6,1$ & $15,7 \pm 7,5$ & $*$ \\
Plaque index & $\mathbf{0 , 7} \pm \mathbf{0 , 3}$ & $\mathbf{1 , 1 3} \pm \mathbf{0 , 6}$ & \\
Bleeding index (\%) & $33,3 \%$ & $38,1 \%$ & $* *$ \\
Periodontal pocket depth (mm) & $\mathbf{3 , 0 9} \pm \mathbf{0 , 6}$ & $\mathbf{3 , 5 8} \pm \mathbf{0 , 5}$ & $* *$ \\
Moderate pockets (\%) & $\mathbf{3 9 , 0} \%$ & $\mathbf{5 7 , 9} \%$ & \\
Attachment loss (mm) & $4,15 \pm 1,11$ & $4,49 \pm 1,0$ & \\
\hline
\end{tabular}

n: sample size.

DMFT: decayed, missing and filled teeth index. $* p<0,05 \quad * * p<0,01$.

The mean number of teeth in the mouth was $20.2 \pm 5.2$ among the patients and $20.2 \pm 6.0$ in the control group. The mean DMFT index was $15.7 \pm 7.5$ in the patients scheduled for heart valve surgery and $14.8 \mathrm{v} 6.1$ in the controls - the difference between groups being no significant ( $p=0.594)$.

The mean dental plaque index was significantly higher in the patient group $(1.13 \pm 0.62$ versus $0.76 \pm 0.39$; $p=0.023$ ).

The bleeding index tended to be greater in the patients with valve disease (38.10\% versus $33.31 \%$ in the controls), though significance was not reached (Student t-test).

The mean pocket depth was significantly greater among the patients $(3.58 \pm 0.53$ versus $3.09 \pm 0.69 \mathrm{~mm}$ in the control group; $p=0.004)$. However, on considering the different depth intervals (physiological, moderate, seve- females and 8 males, with a mean age of $71.5 \pm 9.5$ years), while 14 presented mitral valve stenosis (13 females and one male, with a mean age of $73.1 \pm 6.9$ years).

The number of teeth present in the mouth was similar in both subgroups (19.9 \pm 5.8 in the patients with aortic valve stenosis versus $20.6 \pm 6.4$ in those with mitral valve stenosis). Lastly, the DMFT score was $17.3 \pm 8.2$ in the aortic stenosis subgroup and $14.0 \pm 6.5$ in the mitral valve stenosis subgroup $(p=0.21)$.

There were no differences between the two groups in terms of oral mucosal lesions or the panoramic X-rays findings.

\section{Discussion}

Oral infections may pose a risk during the postoperative period of heart valve surgery (15). It has been seen 
that patients scheduled for cardiovascular surgery have poorer oral hygiene than the general population. The present pilot study was therefore designed to evaluate the presence of periodontal and oral infectious disease in patients before heart valve surgery. No studies of this kind have been carried out to date in Spain, and only limited information is available referred to populations in other countries such as Sweden, North America and Japan $(11,12,16)$.

Our results show oral health in the patients scheduled for heart valve surgery to be slightly poorer than in individuals without valve disease. In effect, the dental plaque scores were higher, reflecting poorer dental hygiene among the cases versus the controls, and this may imply a greater risk of periodontal disease. One-half of the patients scheduled for heart valve surgery were seen to have moderate active periodontitis.

With regard to the homogeneity of the two study groups, the number of remaining teeth in the mouth and smoking habit were very similar in both cases. A direct relationship therefore may be postulated between increased plaque and the presence of a greater number of pockets of moderate depth, as well as greater attachment loss.

One of the greatest differences between groups was referred to dental plaque. We have found no studies specifically describing the amount of dental plaque in patients with heart valve disease. In contrast, the literature contains some studies on the periodontal health of such patients. Terezhalmy et al. (15) found $43.6 \%$ of their sample to have periodontitis, in coincidence with the observations of Nakamura et al. (12), who found almost one-half of the patients with valve disease to have advanced periodontitis. Another study evidenced alveolar bone loss of up to $70 \%$ in patients of this kind (11). These studies did not address the cause of such an increased prevalence of periodontitis, however.

In our series, almost $60 \%$ of the patients scheduled for heart valve surgery suffered periodontitis. This situation would be a consequence of plaque accumulation secondary to poorer oral hygiene, and could favor the appearance of bacteremia following tooth brushing in these individuals (17). Although periodontal bacteria have been isolated from heart valve tissues of patients with periodontitis (18), heart valve colonization by periodontal pathogens does not seem to be as frequent as once believed, in view of the bacterial adhesion and colonization difficulties posed by the high blood flow pressure levels reached in such areas $(19,20)$.

Bacteremia secondary to periodontal infection is known to be one of the primary causes of infectious endocarditis (21). In particular, patients with heart valve disorders are at an increased risk. The American Heart Association therefore recommended dental exploration and the treatment of buccodental disease prior to performing heart surgery (22). Periodontal treatment is advised in patients with ad- vanced periodontitis, followed by root scaling and ultrasound treatment. Those teeth not amenable to treatment and with a poor prognosis should be removed (12).

Aortic and mitral valve degeneration and calcification are known to be related to advanced age, and are characterized by risk factors similar to those found in arteriosclerosis. The possible association between arteriosclerosis - ischemic heart disease and chronic periodontitis has been reported in the literature $(23,24)$. In contrast, the evidence relating periodontitis to heart valve disease has been more limited.

In our series moderate periodontitis was seen to be more prevalent, while the literature describes a greater presence of severe periodontitis. However, previous studies have not been able to demonstrate that dental treatment before heart valve surgery effectively improves survival over the short or middle term $(25,26)$. On the other hand, some authors suggest that there are differences in the oral disease conditions found in patients with aortic valve disease versus those with mitral valve disorders (27). No such differences were observed in our series, since both subgroups (aortic and mitral valve disease) presented similar findings in terms of oral disease and levels of periodontitis.

In conclusion, we consider that patients scheduled for heart valve surgery should receive treatment for periodontitis, where required, avoiding plaque accumulation and improving oral hygiene and thus periodontal health. Such presurgical preventive measures are moreover inexpensive and accessible.

\section{References}

1. Alfieri O, Vahanian A. The year in cardiology 2016: valvular heart disease. Eur Heart J. 2017;38:628-33.

2. Toro R, Mangas A, Gómez F. Calcified aortic valve disease: association with aterosclerosis. Med Clin (Barc). 2011;136:588-93.

3. Gallego Galiana J, López Castellanos G, Gioia F, Ruiz Ortega RA, Cobo Reinoso ME, Manzano Espinosa L. Right heart failure after pacemaker implantation. Med Clin (Barc). 2015;144:550-2.

4. D'Arcy JL, Coffey S, Loudon MA, Kennedy A, Pearson-Stuttard J, Birks J, et al. Large-scale community echocardiographic screening reveals a major burden of undiagnosed valvular heart disease in older people: the OxVALVE Population Cohort Study. Eur Heart J. 2016;37:3515-22.

5. Funakoshi S, Kaji S, Yamamuro A, Tani T, Kinoshita M, Okada $\mathrm{Y}$, et al. Impact of early surgery in the active phase on long-term outcomes in left-sided native valve infective endocarditis. J Thorac Cardivasc Surg. 2011;142:836-42.

6. Machuca G, Segura-Egea JJ, Jimenez-Beato G, Lacalle JR, Bullon P. Clinical indicators of periodontal disease in patients with coronary heart disease: a 10 years longitudinal study. Med Oral Patol Oral Cir Bucal. 2012;17:e569-74.

7. Meurman JH, Qvarnström M, Janket SJ, Nuutinen P. Oral health and health behavior in patients referred for open -heart surgery. Oral Surg Oral Med Oral Pathol Oral Radiol Endod. 2003;95:300-7.

8. Jansson L, Lavstedt S, Frithiof L, Theobald H. Relationship between oral health and mortality in cardiovascular diseases. J Clin Periodontol. 2001;28:762-8.

9. Geerts SO, Legrand V, Charpentier J, Albert A, Rompen EH. Further evidence of the association between periodontal conditions and coronary artery disease. J Periodontol. 2004;75:1274-80. 
10. Toyofuku T, Inoue Y, Kurihara N, Kudo T, Jibiki M, Sugano N, et al. Differential detection rate of periodontopathic bacteria in atherosclerosis. Surg Today. 2011;41:1395-400.

11. Wu GH, Manzon S, Badovinac R, Woo SB. Oral health, dental treatment, and cardiac valve surgery outcomes. Spec Care Dentist. 2008;28:65-72

12. Nakamura Y, Tagusari O, Seike Y, Ito Y, Saito K, Miyamoto R, et al. Prevalence of periodontitis and optimal timing of dental treatment in patients undergoing heart valve surgery. Interact Cardiovasc Thorac Surg. 2011;12:696-700.

13. Lassnig E, Auer J, Weber T, Berent R, Hartl P, Krennmair G, et al. Infection sources in $\mathrm{HNO}$ - and jawbone regions in patients before valve replacement surgery. Herz. 2004;29:317-21.

14. Grice JW, Barrett PT. A note on Cohen's overlapping proportions of normal distributions. Psychol Rep. 2014;115:741-7.

15. Terezhalmy GT, Safadi TJ, Longworth DL, Muehrcke DD. Oral disease burden in patients undergoing prosthetic heart valve implantation. Ann Thorac Surg. 1997;63:402-4.

16. Hakeberg M, Dernevik L, Gatzinsky P, Eklöf C, Kennergren C, Jontell M. The significance of oral health and dental treatment for the postoperative outcome of heart valve surgery. Scand Cardiovasc J. 1999;33:5-8

17. Lockhart PB, Brennan MT, Thornhill M, Michalowicz BS, Noll J, Bahrani-Mougeot FK, et al. Poor oral higiene as a risk factor for infective endocarditis-related bacteremia. J Am Dent Assoc. 2009;140:1238-44.

18. Nakano K, Nemoto H, Nomura R, Inaba H, Yoshioka H, Taniguchi K, et al. Detection of oral bacteria in cardiovascular specimens. Oral Microbiol Immunol. 2009;24:64-8.

19. Raffaelli L, Santangelo R, Falchetti P, Galluccio F, Luciani N, Anselmi A, et al. Examination of periodontal pathogens in stenotic valve specimens and in whole blood samples in patients affected by aortic valve stenosis and chronic periodontitis. In J Immunopathol Pharmacol. 2010;23:561-6.

20. Radwan-Oczko M, Jaworski A, Dus I, Plonek T, Szulc M, Kustrzycki W. Porphyromonas gingivalis in periodontal pockets and heart valves. Virulence. 2014;5:575-80.

21. Nakamura Y, Tagusari O, Seike Y, Ito Y, Saito K, Miyamoto R, et al. Prevalence of periodontitis and optimal timing of dental treatment in patients undergoing heart valve surgery. Interact Cardivasc Thorac Surg. 2011;12:696-700.

22. Baddour LM, Wilson WR, Bayer AS, Fowler VG Jr, Tleyjeh IM, Rybak MJ, et al. Infective Endocarditis in Adults: Diagnosis, Antimicrobial Therapy, and Management of complications: A Scientific Statement for Healthcare Professionals From the American Heart Association. Circulation. 2015;132:1435-86.

23. Janket SJ, Baird AE, Chuang SK, Jones JA. Meta-analysis of periodontal disease and risk of coronary heart disease and stroke. Oral Surg Oral Med Oral Pathol Oral Radiol Endod. 2003;95:559-69.

24. Blaizot A, Vergnes JN, Nuwwareh S, Amar J, Sixou M. Periodontal diseases and cardiovascular events: meta-analysis of observacional studies. Int Dent J. 2009;59:197-209.

25. Deppe H, Auer-Bahrs J, Kolk A, Hall D, Wagenpfeil S. Need for dental treatment following cardiac valve surgery: A clinical study. J Craniomaxillofac Surg. 2007;35:293-301.

26. Bratel J, Kennergren C, Dernevik L, Hakeberg M. Treatment of oral infections prior to heart valve surgery does not improve longterm survival. Swed Dent J. 2011;35:49-55.

27. Krennmair G, Auer J, Krainhöfner M, Piehslinger E. Odontogenic infection sources in patients scheduled for cardiac valve replacement. Oral Health Prev Dent. 2007;5:153-9.

\section{Conflict of Interest}

*The authors declare no potential conflict of interest and no sources of funding regarding the authorship and/or publication of this article. All authors have viewed and agreed to the submission. All patients whose records were used in the study received detailed information about the study and gave their informed consent to take part on the understanding that personal data and records would remain confidential and would only be used for scientific purposes. 